\title{
Summary of balance of payments
}




\section{Current account}

The UK has recorded a current account deficit in every year since 1984. Prior to 1984, the current account recorded a surplus in 1980 to 1983 . Since the last surplus was recorded in 1983, there have been three main phases in the development of the current account. In the first phase, from 1984 to 1989, the current account deficit increased steadily to reach a high of f25.5 billion in 1989 (equivalent to -4.9 per cent of GDP); during the second phase, from 1990 until 1997, the current account deficit declined to a low of $£ 1.0$ billion in 1997; in the third phase, since 1998, the current account deficit has widened sharply. The deficit in 2007, at $\mathrm{f} 52.6$ billion, is the highest recorded in cash terms but only equates to -3.8 per cent of GDP, which is a lower percentage than in both 1988 and 1989.

The profile for the current account has historically followed that of trade in goods, its biggest and most cyclical component. For a while, at the end of the 1990s, that pattern changed, but in recent years the pattern has re-emerged and the increasing

\section{Figure 1.1}

\section{Current account balance}

f billion

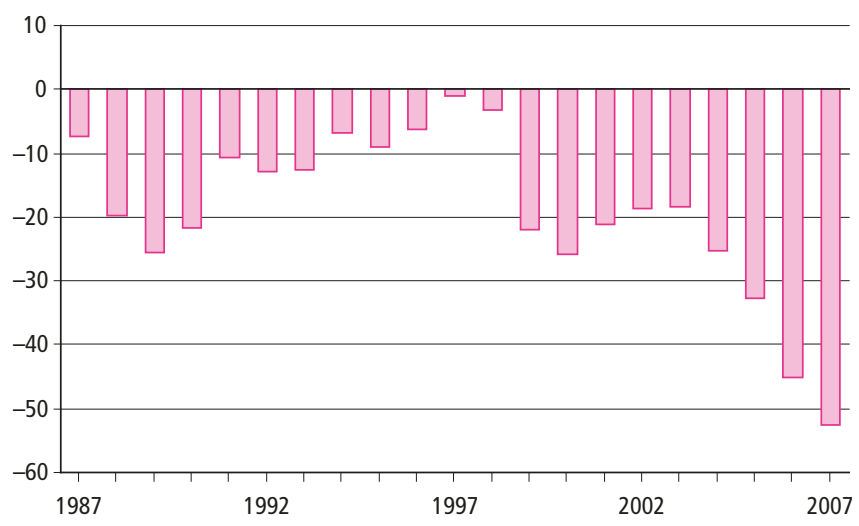

\section{Figure 1.2}

\section{Current balance as a percentage of GDP}

Per cent

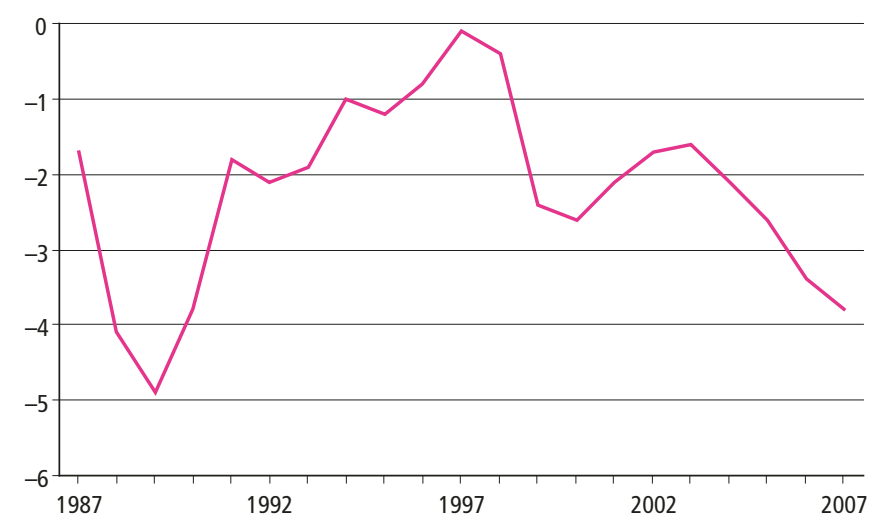

deficit on trade in goods is mirrored by an increase in the current account deficit. The last trade in goods surplus, recorded in 1982, was the main driver of a current account surplus. Following 1982, the goods balance went into deficit and this increased to a peak of $\mathrm{f} 24.7$ billion in 1989 , while the current balance deteriorated to a deficit of $\mathrm{f} 25.5$ billion. From 1989 until the late-1990s, both the trade in goods and current account deficits fell and then subsequently rose. From 1999 to 2003 the goods deficit continued to grow but the current account deficit stabilised, due to a widening income surplus. From 2004, the deficit on trade in goods has increased steadily, matched by a rise in the current account deficit.

\section{Trade in goods and services}

The trade in goods account recorded net surpluses in the years 1980, 1981 and 1982, largely as a result of growth in exports of North Sea oil. Since then, however, the trade in goods account has remained in deficit. The deficit grew significantly in the late 1980s to reach a peak of $f 24.7$ billion in 1989, before narrowing in the 1990s to levels of around $f 10$ billion to $\mathrm{f} 14$ billion. In 1998 the deficit jumped by about $\mathrm{f} 10$ billion, and it has continued to rise since, reaching a cash record of f89.3 billion in 2007.

The trade in services account has shown a surplus for every year since 1966 . The surplus on services increased fairly steadily until 1987 during which time it broadly offset the deficit on trade in goods. From 1988 to 1994 the surplus was reasonably steady at around $£ 7$ billion annually. From 1995 to 1997 the services surplus increased significantly, to around $\mathrm{f} 17$ billion. It dropped back to around $\mathrm{f} 15$ billion in 1998 and began rising again in 2001, reaching $f 28.1$ billion in 2004 . It fell back slightly in 2005 and then rose to a record $\mathrm{f} 41.8$ billion in the latest year.

\section{Figure 1.3}

\section{Trade in goods and services Credits less debits}

$\mathrm{f}$ billion

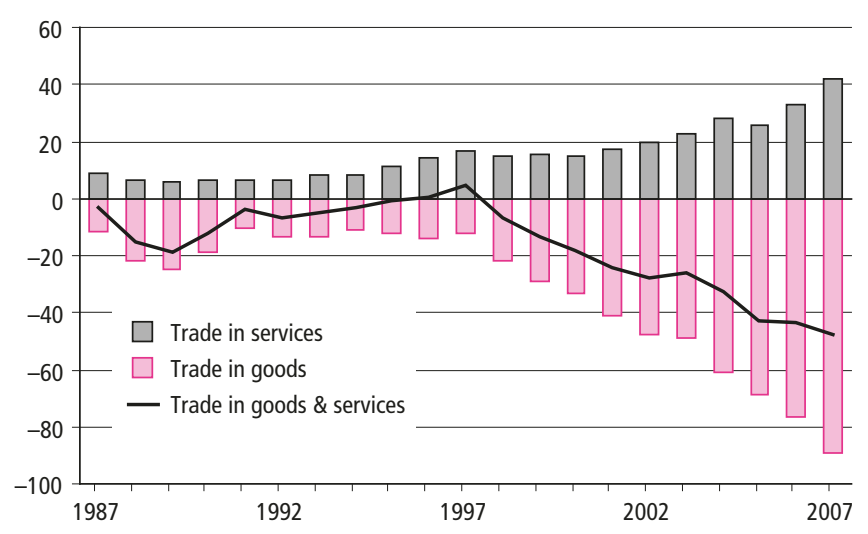




\section{Income}

The income account consists of compensation of employees and investment income, the latter dominating the account. Historically the balance on compensation of employees has generally been in deficit. It moved into surplus in the late 1990s but moved back into deficit in 2004, where it has remained.

The investment income balance was in surplus until 1976. From 1977 to 1996 it showed a deficit in nearly every year. Then from 1997 onwards it has shown a surplus in every year except for 1999. Surpluses on direct investment income have been partly offset by deficits on other investment - principally banks' net interest payments on loans and deposits. From 2002 to 2004 the surplus was around $£ 18$ billion in each year. In 2005 the surplus peaked at $\mathrm{f} 22.5$ billion, due to record net earnings of $\mathrm{f} 43.0$ billion on direct investment, but then dropped sharply in 2006 to $\mathrm{f} 10.8$ billion, decreasing further in 2007 to f9.3 billion. By sector, net earnings of UK monetary financial institutions (banks and building societies) were $£ 9.2$ billion in 2007, compared to $\mathrm{f2.2}$ billion in 2006. However, this increase was outweighed by a $\mathrm{f2.1}$ billion increase in the net payments by central government in 2007 together with a $\mathbf{f 6 . 1}$ billion fall in net earnings by other sectors.

\section{Figure 1.4}

\section{Investment income \\ Credits less debits \\ f billion}

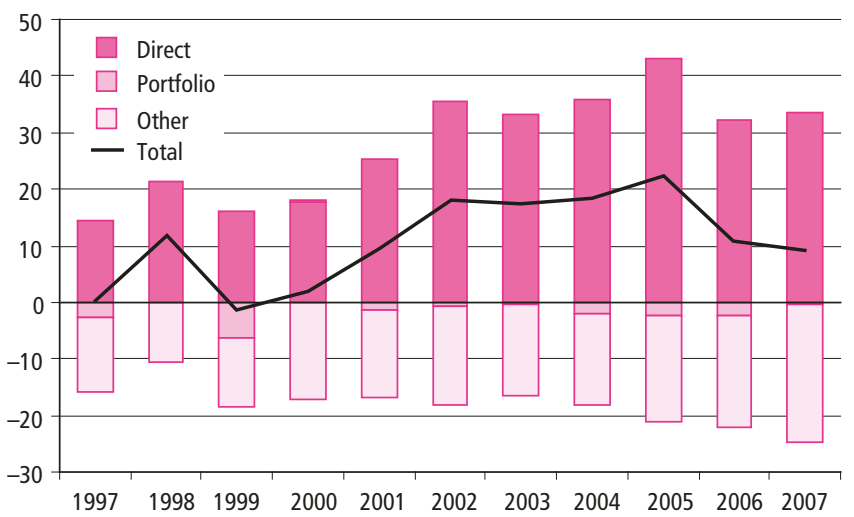

\section{Current transfers}

The transfers account has shown a deficit in every year since 1960. The deficit increased steadily to reach $£ 4.8$ billion in 1990. In 1991, the deficit reduced to $f 1.0$ billion, reflecting f2.1 billion receipts from other countries towards the UK's cost of the first Gulf conflict. The deficit has since increased, to reach a record $\mathrm{f} 13.7$ billion in 2007. Separate data for general government and other sectors are available from 1986 and show that both have been consistently in deficit since 1992 . The majority of payments to and receipts from EU institutions are recorded as other sector transactions as they relate to the original payee or ultimate recipient of the payment/receipt. The volatility in this account is driven by fluctuating net contributions to EU Institutions.

\section{Figure 1.5}

\section{Current transfers Credits less debits}

$\mathrm{f}$ billion

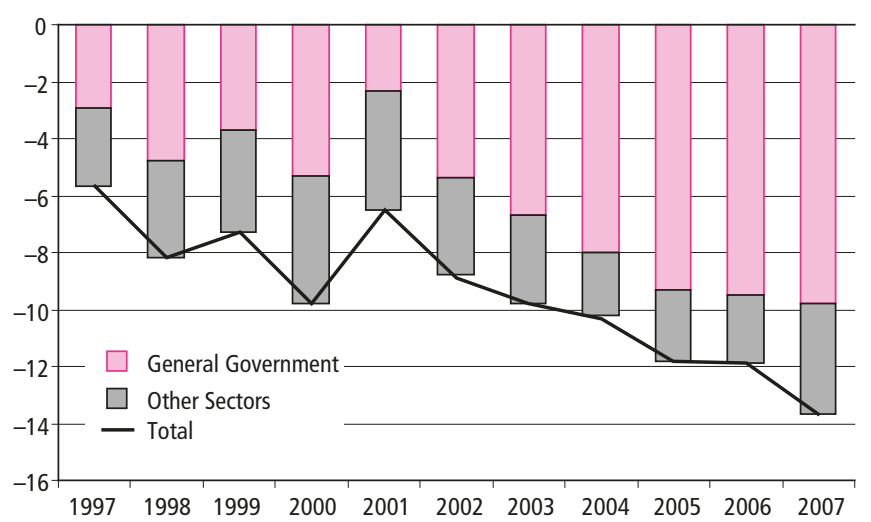

\section{Revisions since Pink Book 2007}

The current account balance has been revised back to 1987 in this publication as compared with data published in Pink Book 2007. However, from 1971, equal and offsetting revisions have been made to the balances for trade in services and for investment income, to move FISIM (financial intermediation services indirectly measured) from investment income to trade in services (see methodological notes for table 3.6). The revisions to the current account balance stem from the inclusion of new data sources and a series of methodological improvements. Details of the sources of these changes are given on pages 17-18 of the Introduction; the impact of the changes can be seen in figure 1.6 and in table 1.1R.

\section{Figure 1.6}

\section{Revisions since Pink Book 2007 Credits less debits \\ f billion}

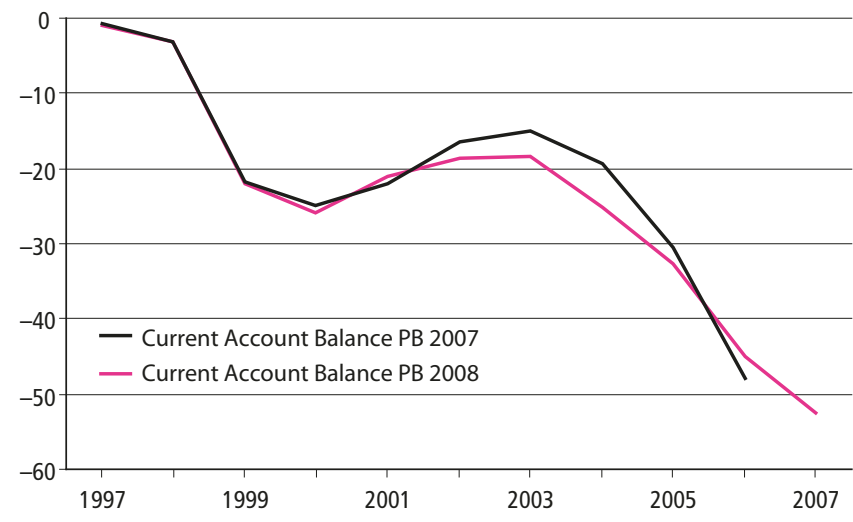




\section{Investment flows, levels and income}

One important set of relationships within the balance of payments is the link between the financial account (investment flows), the international investment position (levels or balance sheets), and the income deriving from the balance sheets. This is explained in more detail in the Introduction. Although a reconciliation statement between opening and closing levels and flows is not officially compiled in the UK, table 1.3 shows the rudiments of this relationship over the years for which consistent detailed data are available. Within the three main categories of investment (direct, portfolio and other), as well as reserve assets, it can be seen that the difference in the values of the balance sheet at the end of one year and the previous year is approximately equal to the value of financial transactions in that year. The difference between the two amounts is explained by valuation, exchange rate and other effects, for example, company write-offs, etc.

The value of both external assets and liabilities in the international investment position has been rising steadily since 1980, reflecting both the increased global investment and the increasing prices of external assets and liabilities. Except for 1990, the UK's external assets exceeded external liabilities in every year until 1995; however, since then external liabilities have exceeded external assets. Since 1995, there has been a more than fourfold increase in both the levels of external assets and liabilities with the latter reaching $\mathrm{f} 6.7$ trillion at the end of 2007. Since 2004, the net liability position has increased steadily to end 2007 at $£ 381.6$ billion.

\section{Figure 1.7}

\section{International investment position and income Credits less debits}

f billion (IIP)

$\mathrm{f}$ billion (income)

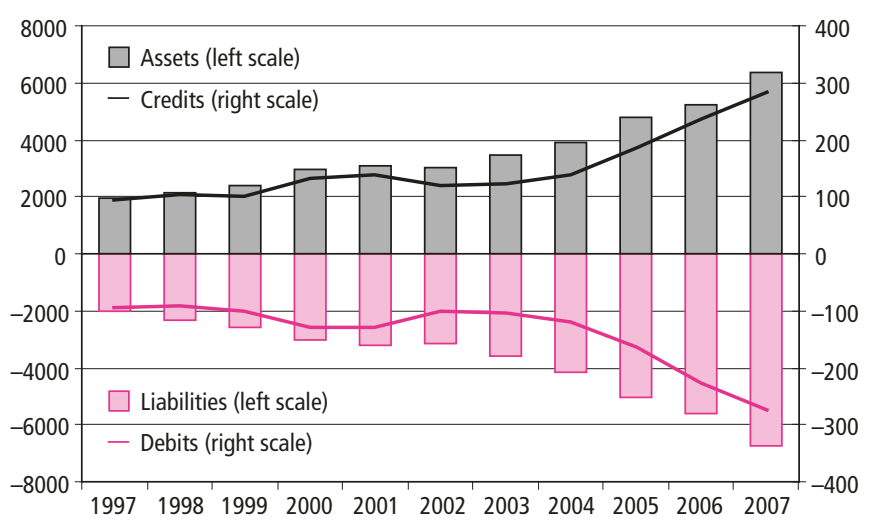

\section{Implied "rates of return"}

Another important relationship is that which exists between investment income and the international investment position. This can be considered most easily by looking at the implied 'rates of return' for both assets and liabilities. In total the implied rate of return on liabilities was higher than assets from the late 1970s until the mid 1990s. Since 1997, although the return on assets has been higher, both have been at relatively low levels. Because other investment constitutes around half of the value of the balance sheets it is not surprising that the rates of return have reflected the movements in interest rates on loans and deposits such as LIBOR.

The rates of return for direct investment are significantly higher than for other forms of investment. This is probably a consequence in part of comparatively lower valuations since direct investment levels are at book value rather than market value used elsewhere, but may also reflect the higher return required to make the longer term investment worthwhile.

Within portfolio investment, rates of return on debt securities are higher than on equity securities. This difference narrowed from 2000 to 2003 but has steadily widened again since then.

Rates of return on other investment were similar to returns on debt in the early 1990s. Between then and 2004 they were, in general, in decline. However, from 2005 onwards they have increased.

\section{Figure $\mathbf{1 . 8}$}

\section{Implied rates of return on assets} Per cent

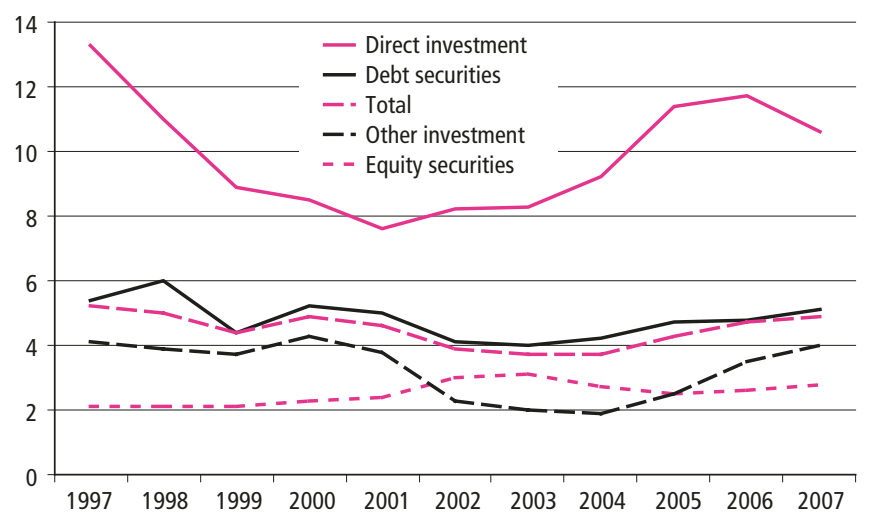

\section{Figure 1.9}

\section{Implied rates of return on liabilities}

Per cent

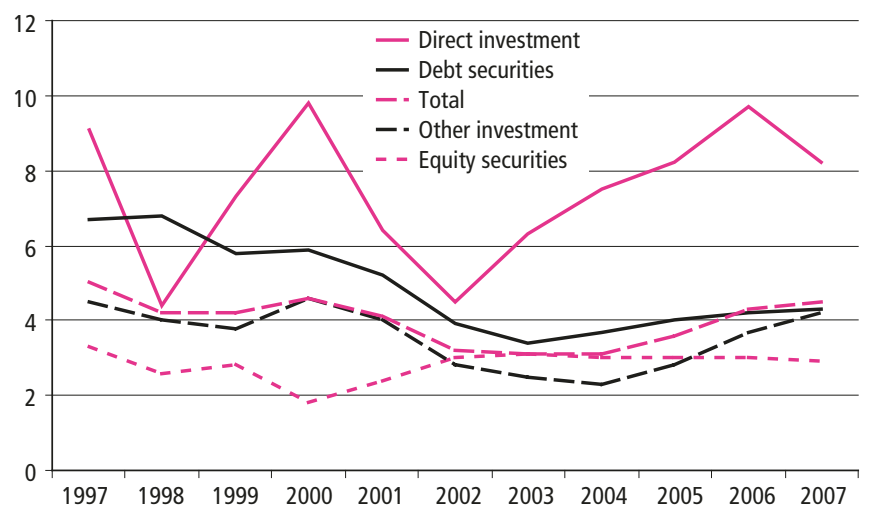


Summary of balance of payments

Balances (credits less debits)

$£$ million

\begin{tabular}{|c|c|c|c|c|c|c|c|c|c|c|c|c|}
\hline & \multicolumn{9}{|c|}{ Current account } & \multirow[b]{2}{*}{$\begin{array}{r}\text { Capital } \\
\text { account }\end{array}$} & \multirow[b]{2}{*}{$\begin{array}{r}\text { Financial } \\
\text { account }\end{array}$} & \multirow[b]{2}{*}{$\begin{array}{r}\text { Net errors } \\
\& \text { omissions }\end{array}$} \\
\hline & $\begin{array}{r}\text { Trade in } \\
\text { goods }\end{array}$ & $\begin{array}{l}\text { Trade in } \\
\text { services }\end{array}$ & $\begin{array}{r}\text { Total goods } \\
\text { and } \\
\text { services }\end{array}$ & $\begin{array}{r}\text { Compensati- } \\
\text { on of } \\
\text { employees }\end{array}$ & $\begin{array}{r}\text { Investment } \\
\text { income }\end{array}$ & $\begin{array}{r}\text { Total } \\
\text { income }\end{array}$ & $\begin{array}{l}\text { Current } \\
\text { transfers }\end{array}$ & $\begin{array}{l}\text { Current } \\
\text { balance }\end{array}$ & $\begin{array}{r}\text { Current } \\
\text { balance as } \\
\% \text { of } G D P^{1}\end{array}$ & & & \\
\hline $\begin{array}{l}1946 \\
1947 \\
1948 \\
1949 \\
1950\end{array}$ & $\begin{array}{r}\text { LQCT } \\
-101 \\
-358 \\
-152 \\
-137 \\
-54\end{array}$ & $\begin{array}{r}\text { KTMS } \\
-274 \\
-197 \\
-64 \\
-43 \\
-4\end{array}$ & $\begin{array}{r}\text { KTMY } \\
-375 \\
-555 \\
-216 \\
-180 \\
-58\end{array}$ & $\begin{array}{r}\text { KTMP } \\
-20 \\
-19 \\
-20 \\
-20 \\
-21\end{array}$ & $\begin{array}{r}\text { HMBM } \\
76 \\
140 \\
223 \\
206 \\
378\end{array}$ & $\begin{array}{r}\text { HMBP } \\
56 \\
121 \\
203 \\
186 \\
357\end{array}$ & $\begin{array}{r}\text { KTNF } \\
166 \\
123 \\
96 \\
29 \\
39\end{array}$ & $\begin{array}{r}\text { HBOG } \\
-153 \\
-311 \\
83 \\
35 \\
338\end{array}$ & $\begin{array}{r}\text { AA6H } \\
. . \\
. .7 \\
0.7 \\
0.3 \\
2.6\end{array}$ & $\begin{array}{r}\text { FKMJ } \\
-21 \\
-21 \\
-17 \\
-12 \\
-10\end{array}$ & $\begin{array}{r}\text { HBNT } \\
181 \\
552 \\
-58 \\
-103 \\
-447\end{array}$ & $\begin{array}{r}\mathrm{HHDH} \\
-7 \\
-220 \\
-8 \\
80 \\
119\end{array}$ \\
\hline $\begin{array}{l}1951 \\
1952 \\
1953 \\
1954 \\
1955\end{array}$ & $\begin{array}{l}-692 \\
-272 \\
-244 \\
-210 \\
-315\end{array}$ & $\begin{array}{r}32 \\
123 \\
123 \\
115 \\
42\end{array}$ & $\begin{array}{r}-660 \\
-149 \\
-121 \\
-95 \\
-273\end{array}$ & $\begin{array}{l}-21 \\
-22 \\
-25 \\
-27 \\
-27\end{array}$ & $\begin{array}{l}322 \\
231 \\
207 \\
227 \\
149\end{array}$ & $\begin{array}{l}301 \\
209 \\
182 \\
200 \\
122\end{array}$ & $\begin{array}{r}29 \\
169 \\
143 \\
55 \\
43\end{array}$ & $\begin{array}{r}-330 \\
229 \\
204 \\
160 \\
-108\end{array}$ & $\begin{array}{r}-2.3 \\
1.4 \\
1.2 \\
0.9 \\
-0.6\end{array}$ & $\begin{array}{l}-15 \\
-15 \\
-13 \\
-13 \\
-15\end{array}$ & $\begin{array}{r}426 \\
-229 \\
-177 \\
-174 \\
34\end{array}$ & $\begin{array}{r}-81 \\
15 \\
-14 \\
27 \\
89\end{array}$ \\
\hline $\begin{array}{l}1956 \\
1957 \\
1958 \\
1959 \\
1960\end{array}$ & $\begin{array}{r}50 \\
-29 \\
34 \\
-116 \\
-404\end{array}$ & $\begin{array}{r}26 \\
121 \\
119 \\
118 \\
39\end{array}$ & $\begin{array}{r}76 \\
92 \\
153 \\
2 \\
-365\end{array}$ & $\begin{array}{l}-30 \\
-32 \\
-34 \\
-37 \\
-35\end{array}$ & $\begin{array}{l}203 \\
223 \\
261 \\
233 \\
201\end{array}$ & $\begin{array}{l}173 \\
191 \\
227 \\
196 \\
166\end{array}$ & $\begin{array}{r}2 \\
-5 \\
4 \\
-6\end{array}$ & $\begin{array}{r}251 \\
278 \\
384 \\
198 \\
-205\end{array}$ & $\begin{array}{r}1.2 \\
1.3 \\
1.7 \\
0.8 \\
-0.8\end{array}$ & $\begin{array}{r}-13 \\
-13 \\
-10 \\
-5 \\
-6\end{array}$ & $\begin{array}{r}-250 \\
-313 \\
-411 \\
-68 \\
-7\end{array}$ & $\begin{array}{r}12 \\
48 \\
37 \\
-125 \\
218\end{array}$ \\
\hline $\begin{array}{l}1966 \\
1967 \\
1968 \\
1969 \\
1970\end{array}$ & $\begin{array}{r}-111 \\
-601 \\
-708 \\
-214 \\
-18\end{array}$ & $\begin{array}{r}44 \\
157 \\
341 \\
392 \\
455\end{array}$ & $\begin{array}{r}-67 \\
-444 \\
-367 \\
178 \\
437\end{array}$ & $\begin{array}{l}-39 \\
-39 \\
-48 \\
-47 \\
-56\end{array}$ & $\begin{array}{l}358 \\
354 \\
303 \\
468 \\
527\end{array}$ & $\begin{array}{l}319 \\
315 \\
255 \\
421 \\
471\end{array}$ & $\begin{array}{r}-91 \\
-118 \\
-119 \\
-109 \\
-89\end{array}$ & $\begin{array}{r}161 \\
-247 \\
-231 \\
490 \\
819\end{array}$ & $\begin{array}{r}0.4 \\
-0.6 \\
-0.5 \\
1.0 \\
1.6\end{array}$ & $\begin{array}{l}-19 \\
-25 \\
-26 \\
-23 \\
-22\end{array}$ & $\begin{array}{r}22 \\
179 \\
688 \\
-794 \\
-818\end{array}$ & $\begin{array}{r}-164 \\
93 \\
-431 \\
327 \\
21\end{array}$ \\
\hline $\begin{array}{l}1971 \\
1972 \\
1973 \\
1974 \\
1975\end{array}$ & $\begin{array}{r}205 \\
-736 \\
-2573 \\
-5241 \\
-3245\end{array}$ & $\begin{array}{r}617 \\
722 \\
907 \\
1292 \\
1708\end{array}$ & $\begin{array}{r}822 \\
-14 \\
-1666 \\
-3949 \\
-1537\end{array}$ & $\begin{array}{r}-63 \\
-52 \\
-68 \\
-92 \\
-102\end{array}$ & $\begin{array}{r}454 \\
350 \\
970 \\
1010 \\
257\end{array}$ & $\begin{array}{l}391 \\
298 \\
902 \\
918 \\
155\end{array}$ & $\begin{array}{r}-90 \\
-142 \\
-336 \\
-302 \\
-313\end{array}$ & $\begin{array}{r}1123 \\
142 \\
-1100 \\
-3333 \\
-1695\end{array}$ & $\begin{array}{r}2.0 \\
0.2 \\
-1.5 \\
-4.0 \\
-1.6\end{array}$ & $\begin{array}{l}-23 \\
-35 \\
-39 \\
-34 \\
-36\end{array}$ & $\begin{array}{r}-1330 \\
477 \\
1031 \\
3185 \\
1569\end{array}$ & $\begin{array}{r}230 \\
-584 \\
108 \\
182 \\
162\end{array}$ \\
\hline $\begin{array}{l}1976 \\
1977 \\
1978 \\
1979 \\
1980\end{array}$ & $\begin{array}{r}-3930 \\
-2271 \\
-1534 \\
-3326 \\
1329\end{array}$ & $\begin{array}{l}2872 \\
3704 \\
4215 \\
4573 \\
4414\end{array}$ & $\begin{array}{r}-1058 \\
1433 \\
2681 \\
1247 \\
5743\end{array}$ & $\begin{array}{r}-140 \\
-152 \\
-140 \\
-130 \\
-82\end{array}$ & $\begin{array}{r}760 \\
-678 \\
-300 \\
-342 \\
-2268\end{array}$ & $\begin{array}{r}620 \\
-830 \\
-440 \\
-472 \\
-2350\end{array}$ & $\begin{array}{r}-534 \\
-889 \\
-1420 \\
-1777 \\
-1653\end{array}$ & $\begin{array}{r}-972 \\
-286 \\
821 \\
-1002 \\
1740\end{array}$ & $\begin{array}{r}-0.8 \\
-0.2 \\
0.5 \\
-0.5 \\
0.8\end{array}$ & $\begin{array}{r}-12 \\
11 \\
-79 \\
-103 \\
-4\end{array}$ & $\begin{array}{r}507 \\
-3286 \\
-2655 \\
864 \\
-2157\end{array}$ & $\begin{array}{r}477 \\
3561 \\
1913 \\
241 \\
421\end{array}$ \\
\hline $\begin{array}{l}1986 \\
1987 \\
1988 \\
1989 \\
1990\end{array}$ & $\begin{array}{r}-9617 \\
-11698 \\
-21553 \\
-24724 \\
-18707\end{array}$ & $\begin{array}{l}8182 \\
8604 \\
6388 \\
5566 \\
6643\end{array}$ & $\begin{array}{r}-1435 \\
-3094 \\
-15165 \\
-18858 \\
-12064\end{array}$ & $\begin{array}{r}-156 \\
-174 \\
-64 \\
-138 \\
-110\end{array}$ & $\begin{array}{r}71 \\
-730 \\
-1188 \\
-2309 \\
-4586\end{array}$ & $\begin{array}{r}-85 \\
-904 \\
-1252 \\
-2447 \\
-4696\end{array}$ & $\begin{array}{l}-2094 \\
-3437 \\
-3293 \\
-4228 \\
-4802\end{array}$ & $\begin{array}{r}-3614 \\
-7435 \\
-19710 \\
-25533 \\
-21562\end{array}$ & $\begin{array}{l}-0.9 \\
-1.7 \\
-4.1 \\
-4.9 \\
-3.8\end{array}$ & $\begin{array}{l}135 \\
333 \\
235 \\
270 \\
497\end{array}$ & $\begin{array}{r}-122 \\
10606 \\
16989 \\
13614 \\
22272\end{array}$ & $\begin{array}{r}3601 \\
-3504 \\
2486 \\
11649 \\
-1207\end{array}$ \\
\hline $\begin{array}{l}1991 \\
1992 \\
1993 \\
1994 \\
1995\end{array}$ & $\begin{array}{l}-10223 \\
-13050 \\
-13066 \\
-11 \quad 126 \\
-12023\end{array}$ & $\begin{array}{rl}6 & 312 \\
6 & 353 \\
8 & 174 \\
8 & 161 \\
11 & 165\end{array}$ & $\begin{array}{r}-3911 \\
-6697 \\
-4892 \\
-2965 \\
-858\end{array}$ & $\begin{array}{r}-63 \\
-49 \\
35 \\
-170 \\
-296\end{array}$ & $\begin{array}{r}-5642 \\
-1037 \\
-2547 \\
1521 \\
-546\end{array}$ & $\begin{array}{r}-5705 \\
-1086 \\
-2512 \\
1351 \\
-842\end{array}$ & $\begin{array}{r}-999 \\
-5228 \\
-5056 \\
-5187 \\
-7363\end{array}$ & $\begin{array}{r}-10615 \\
-13011 \\
-12460 \\
-6801 \\
-9063\end{array}$ & $\begin{array}{l}-1.8 \\
-2.1 \\
-1.9 \\
-1.0 \\
-1.2\end{array}$ & $\begin{array}{r}290 \\
421 \\
309 \\
33 \\
533\end{array}$ & $\begin{array}{r}7855 \\
16311 \\
22278 \\
-3240 \\
-1717\end{array}$ & $\begin{array}{r}2470 \\
-3721 \\
-10127 \\
10008 \\
10247\end{array}$ \\
\hline $\begin{array}{l}1996 \\
1997 \\
1998 \\
1999 \\
2000\end{array}$ & $\begin{array}{l}-13722 \\
-12342 \\
-21813 \\
-29051 \\
-32976\end{array}$ & $\begin{array}{l}14312 \\
16801 \\
15003 \\
15562 \\
15002\end{array}$ & $\begin{array}{r}590 \\
4459 \\
-6810 \\
-13489 \\
-17974\end{array}$ & $\begin{array}{r}93 \\
83 \\
-10 \\
201 \\
150\end{array}$ & $\begin{array}{r}-2460 \\
241 \\
11813 \\
-1244 \\
1812\end{array}$ & $\begin{array}{r}-2367 \\
324 \\
11803 \\
-1043 \\
1962\end{array}$ & 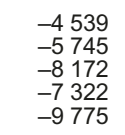 & $\begin{array}{r}-6316 \\
-962 \\
-3179 \\
-21854 \\
-25787\end{array}$ & $\begin{array}{l}-0.8 \\
-0.1 \\
-0.4 \\
-2.4 \\
-2.6\end{array}$ & $\begin{array}{r}1260 \\
958 \\
489 \\
747 \\
1703\end{array}$ & $\begin{array}{r}-940 \\
-7294 \\
4480 \\
29505 \\
23133\end{array}$ & $\begin{array}{r}5996 \\
7298 \\
-1790 \\
-8398 \\
951\end{array}$ \\
\hline $\begin{array}{l}2001 \\
2002 \\
2003 \\
2004 \\
2005\end{array}$ & $\begin{array}{l}-41212 \\
-47705 \\
-48607 \\
-60900 \\
-68589\end{array}$ & $\begin{array}{l}17200 \\
19632 \\
22612 \\
28146 \\
25875\end{array}$ & $\begin{array}{l}-24012 \\
-28073 \\
-25995 \\
-32754 \\
-42714\end{array}$ & $\begin{array}{r}66 \\
67 \\
59 \\
-494 \\
-610\end{array}$ & $\begin{array}{r}9359 \\
18219 \\
17464 \\
18324 \\
22482\end{array}$ & $\begin{array}{r}9425 \\
18286 \\
17523 \\
17830 \\
21872\end{array}$ & $\begin{array}{r}-6515 \\
-8870 \\
-9835 \\
-10276 \\
-11849\end{array}$ & $\begin{array}{l}-21102 \\
-18657 \\
-18307 \\
-25200 \\
-32691\end{array}$ & $\begin{array}{l}-2.1 \\
-1.7 \\
-1.6 \\
-2.1 \\
-2.6\end{array}$ & $\begin{array}{r}1318 \\
932 \\
1466 \\
2064 \\
1503\end{array}$ & $\begin{array}{l}27194 \\
24204 \\
22553 \\
19564 \\
30581\end{array}$ & $\begin{array}{r}-7410 \\
-6479 \\
-5712 \\
3572 \\
607\end{array}$ \\
\hline $\begin{array}{l}2006 \\
2007\end{array}$ & $\begin{array}{l}-76313 \\
-89252\end{array}$ & $\begin{array}{l}33108 \\
41772\end{array}$ & $\begin{array}{l}-43204 \\
-47480\end{array}$ & $\begin{array}{l}-745 \\
-665\end{array}$ & $\begin{array}{r}10842 \\
9271\end{array}$ & $\begin{array}{r}10097 \\
8606\end{array}$ & $\begin{array}{l}-11924 \\
-13694\end{array}$ & $\begin{array}{l}-45031 \\
-52568\end{array}$ & $\begin{array}{l}-3.4 \\
-3.8\end{array}$ & $\begin{array}{r}975 \\
2641\end{array}$ & $\begin{array}{l}41875 \\
39830\end{array}$ & $\begin{array}{r}2181 \\
10097\end{array}$ \\
\hline
\end{tabular}

1 Using series YBHA: GDP at current market prices. 


\section{D Summary of balance of payments \\ Revisions since ONS Pink Book 2007}

$£$ million

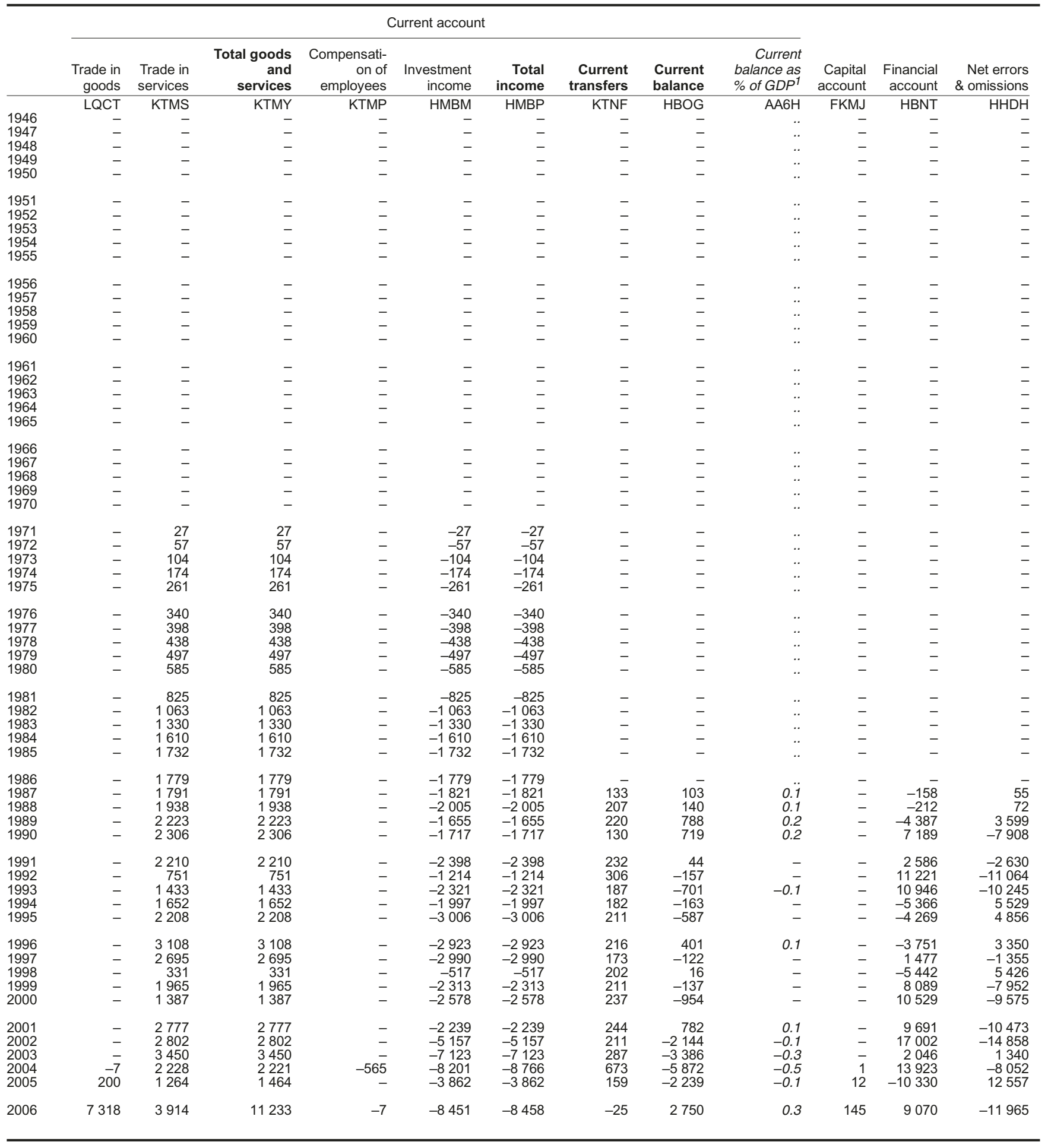

1 Using series YBHA: GDP at current market prices. 
$£$ million

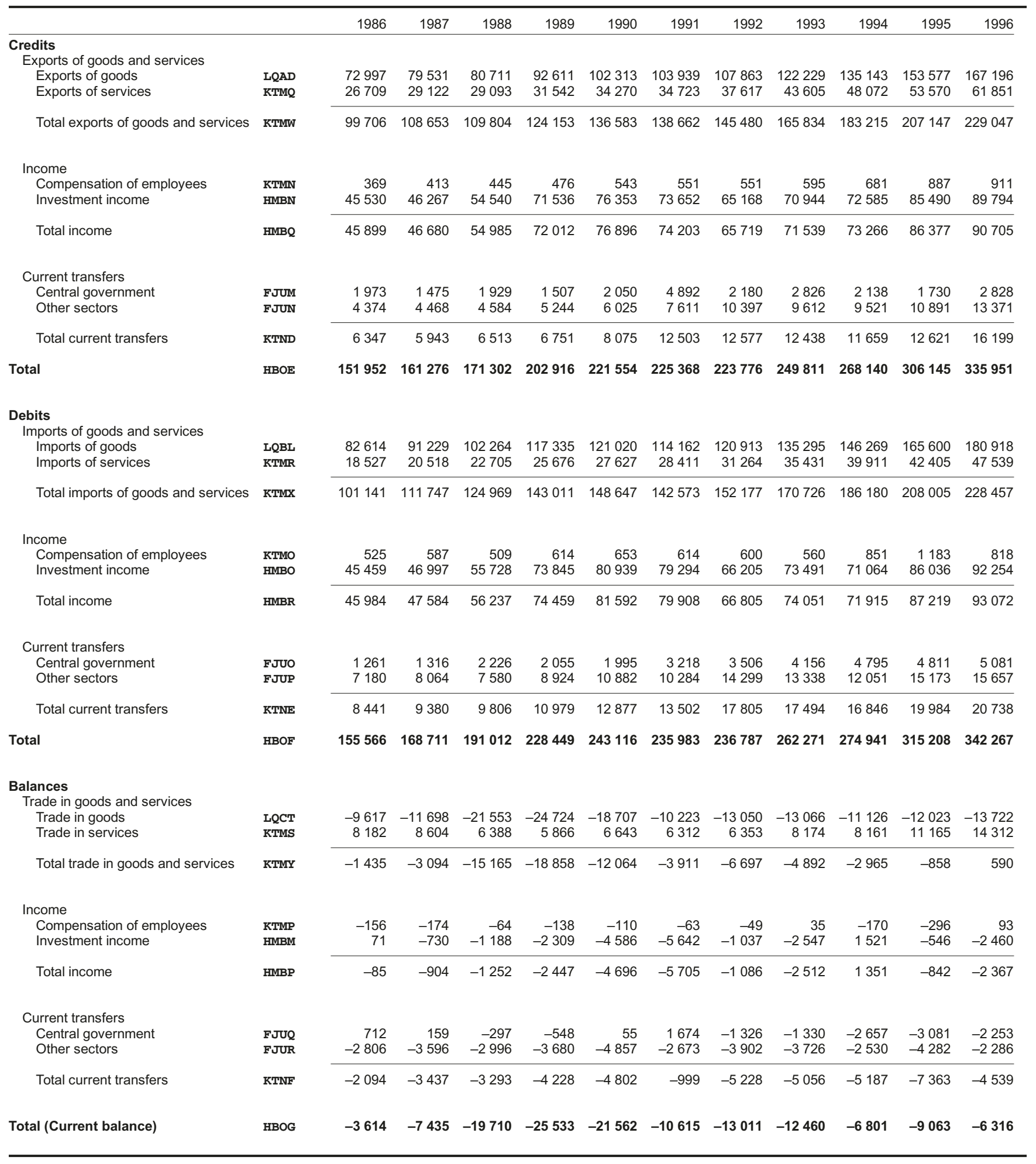




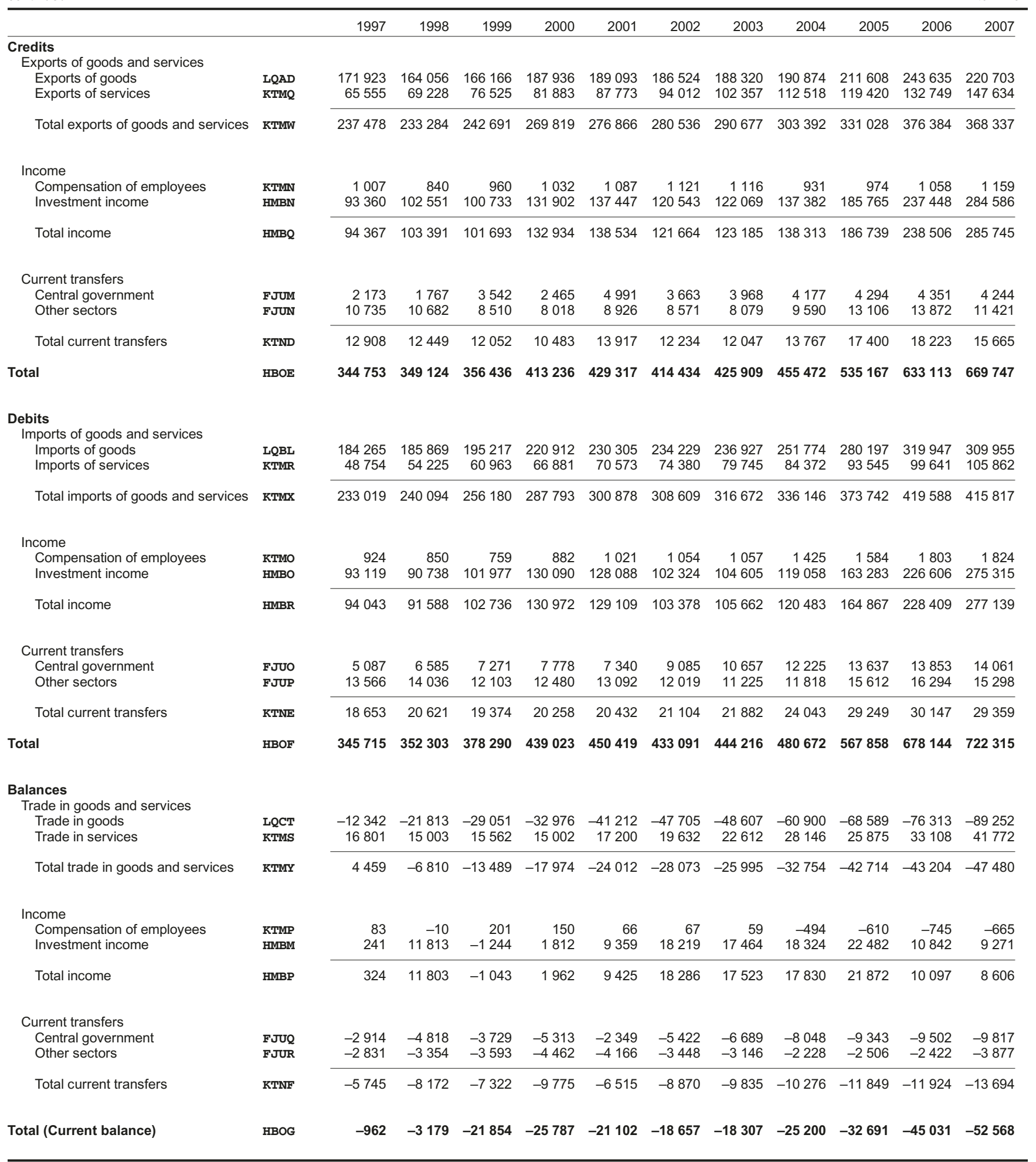




\section{Summary of international investment position, financial account - -3 and investment income}

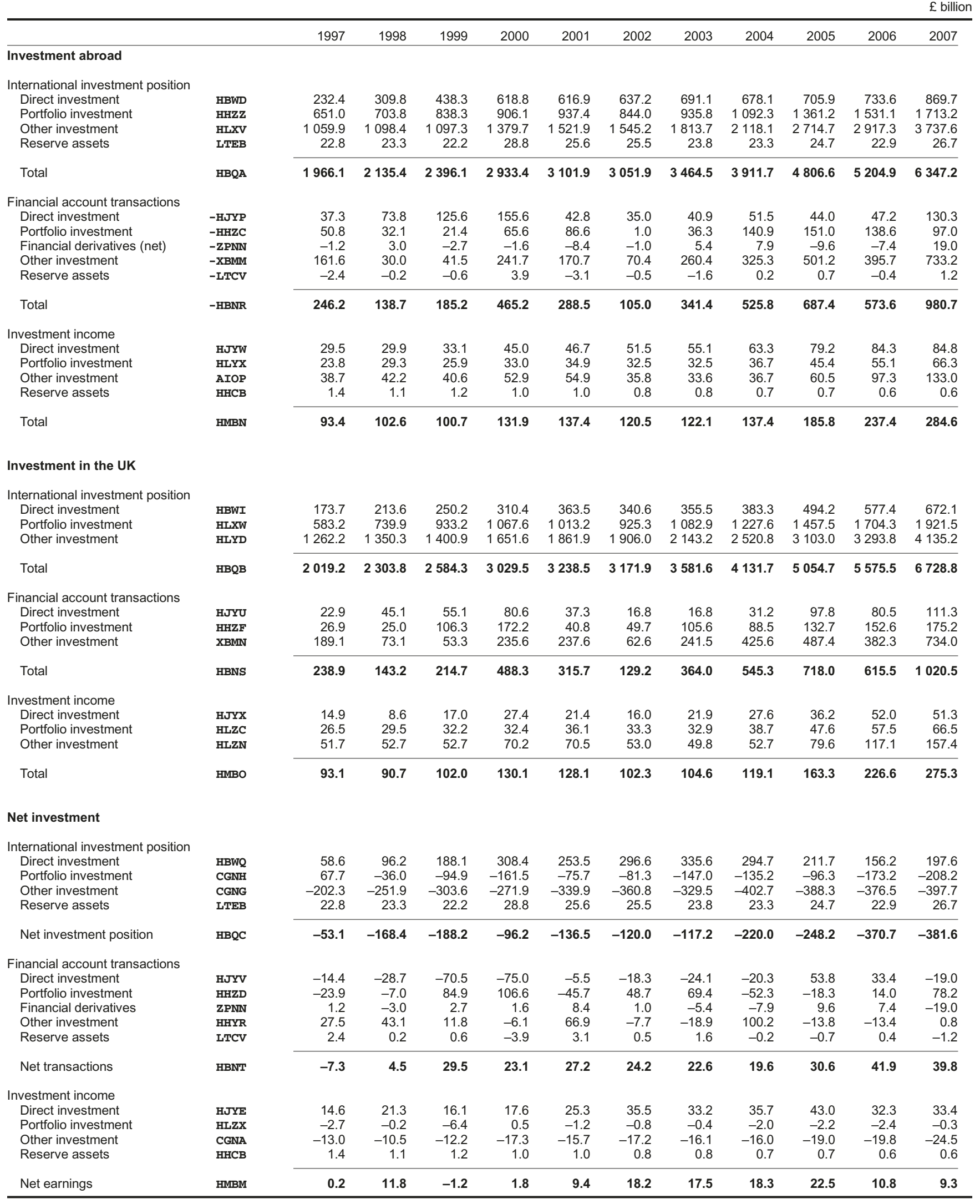

